Schirrmeister, Albert: »Folter und Heiligung in der $>$ Legenda Aurea $<$. Frühchristliche Martern und spätmittelalterliche Körperkonzepte«, in: P. Burschel/G. Distelrath/S. Lembke (Hg.), Das Quälen des Körpers. Eine historische Anthropologie der Folter, Köln: Böhlau Verlag 2000, S. 133-149.

Touber, Anthonius H. (Hg.): Das Donaueschinger Passionsspiel, nach d. Handschrift, mit Einl. u. Kommentar neu hg. von Anthonius H. Touber, Stuttgart: Reclam 1985.

Voragine, Jacobus de: Legenda aurea, ausgewählt, übersetzt und herausgegeben von Rainer Nickel, Stuttgart: Reclam 1988.

- ders.: Die Legenda aurea, aus dem Lat. übers. von Richard Benz, 14. Aufl., Gütersloh: Gütersloher Verlags-Haus 2004.

Warning, Rainer: Funktion und Struktur. Die Ambivalenzen des geistlichen Spiels, München: Fink 1974 (Theorie und Geschichte der Literatur und der schönen Künste; 35).

Wolf, Klaus: Kommentar zur $»$ Frankfurter Dirigierrolle« und zum $»$ Frankfurter Passionsspiel«, Tübingen: Niemeyer 2002.Körper und Welt.

\title{
Die Gewalt des Namens. \\ Von der Missachtung zum sozialen Tod
}

STEFFEN K. HERRMANN

»Im Konzentrationslager waren wir keine Menschen, sondern nur Nummern! Meine Lagernummer war 45 708.«

Ilse Stephan

》66 903. Das ist sehr - ich kann meine Nummer immer nur auf deutsch sagen. Ich war eine Nummer, ich war doch keine Person. $\ll^{2}$

Varda Z.

»Ein weiblicher Häftling von der politischen Abteilung mit einer sehr niedrigen Nummer [...] ergriff meine Hand und begann, die nächste Nummer einzustechen [...] Ich fühlte, dass sie mich eigentlich nicht in den Arm, sondern

ins Herz stach. Von diesem Augenblick an hatte ich aufgehört, ein Mensch zu sein. Ich hörte auf zu fühlen, zu denken. Ich besaß keinen Namen, keine Adresse mehr. Ich war Häftling Nr. 55 908.

Krystina Zywulska

Ein zentrales Ritual bei der Einlieferung in das Konzentrationslager Auschwitz war die Eintätowierung der Nummer und der Entzug des Namens. Von vielen überlebenden Häftlingen wissen wir, dass dieser Akt als besonders einschneidendes Erlebnis empfunden wurde. Das hat seinen Grund darin, dass der Name ein besonderes Zeichen ist: Durch ihn werden wir nicht nur einzigartig und unverwechselbar, sondern zugleich auch als soziale Wesen, die symbolisch

I Hae Henneherg (Hg.): Vem Nemmen zull Nummer. Einlieferungsritual in

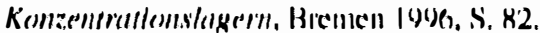

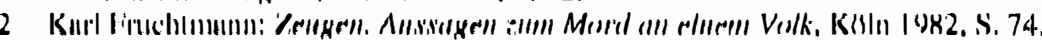

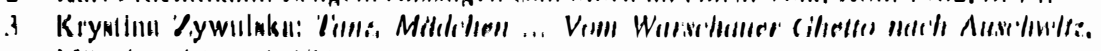

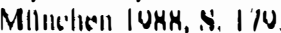


adressierbar sind, allererst hervorgebracht. Das hat allerdings auch zur Folge, dass im Akt der Namensgebung eine der tiefgreifendsten Formen der symbolischen Verletzbarkeit wurzelt. Diese ist in der Geschichte des Antisemitismus auf einzigartige Weise zum Ziel einer Politik des Eigennamens geworden. Was sich zunächst in Spott- und Schimpfnamen noch als scheinbar harmlose und alltägliche Stichelei gegenüber Jüdinnen und Juden zeigte, entfaltete seine Gewalt in einer systematischen, staatlich initiierten Politik des Eigennamens, die zur Demütigung und Ausschließung einer ganzen Bevölkerungsgruppe diente und gipfelte in den Konzentrationslagern, in denen die Nummer zum Stellvertreter des menschlichen Namens wurde. In der Geschichte des jüdischen Namens spiegeln sich derart nicht nur verschiedene Stufen des Antisemitismus wieder, sondern ebenso zeigen sich in dieser Geschichte auch ganz unterschiedliche Formen einer namentlichen Verletzungsmacht

In der philosophischen Debatte wurde die Verletzbarkeit durch den Namen bisher fast vollständig vernachlässigt. Die anhaltenden Diskussionen um den Eigennamen haben zwar eine lange Tradition, doch sie orientieren sich zumeist allein an semantischen und referentiellen Wahrheitsfragen. ${ }^{4}$ Der soziale Sinn des Namens dagegen, was es heißt, einen Namen zu tragen, welche Handlungsmacht in ihm liegt und welche Formen der Verletzbarkeit damit einhergehen, ist bisher nahezu ausnahmslos unbeachtet geblieben. Doch gerade die Möglichkeit, mit den Namen etwas zu tun, seine performative Handlungsdimension also, muss in den Vordergrund treten, um die Rolle des Eigennamens in sozialen Ausschlussprozessen zu verstehen. Ausgehend von der Tatsache des Angesprochenwerdens soll daher im Folgenden vor allem die soziale Dimension des Eigennamens reflektiert werden. Von hier aus lässt sich nicht nur verstehen, warum der jüdische Name im NS zum Ziel einer systematischen Namenspolitik wurde, sondern zugleich auch lassen sich unterschiedliche Fluchtlinien der Verletzungskraft des Namens herausarbeiten, die von der einfachen Missachtung bis hin zur Entmenschlichung reichen

\section{In der Geborgenheit des Daseins}

Am Anfang des Sprechens steht das Grüßen: Die Ansprache zwischen zwei Menschen beginnt meist mit Worten wie >Guten Tag $<$, >Hallo< oder $>$ Willkommen<, die zur Eröffnung eines Gespräches dienen. Diese gesprächsstiftende Funktion des Grußes findet sich auch in der Etymologie des mittelhochdeutschen >gruozen $<$, das auf ein ganz wörtliches >zum Sprechen bringen $<$ und

4 Stellvertretend sei hier der Sammelband von (J'sula Wolf (Hg.): Hilgemmumern.

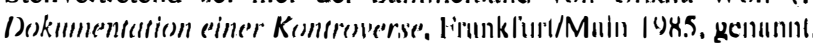

$>$ Reden machen< verweist. ${ }^{5}$ Die Begrüßung selbst bietet dabei jedoch noch keinen Gesprächsstoff, sie hat kaum einen semantischen Gehalt, sie bereitet vielmehr den inhaltlichen Austausch allererst vor. Erving Goffman sieht in der Begrüßung daher vor allem ein $>$ Zugänglichkeitsritual $<$ und verweist darauf, dass die Frage »Wie geht's? « innerhalb eines Begrüßungsrituals normalerweise keine wörtliche Funktion hat und daher auch keiner Antwort bedarf bzw. mit einem obligatorischen »Danke« oder einfach mit der Gegenfrage »Wie geht's?« beantwortet werden kann. ${ }^{6}$ Dass die Begrüßung nichts im wörtlichen Sinne sagt, heißt jedoch nicht, dass sie belanglos wäre - vielmehr ist in ihr schon ein entscheidender sozialer Aspekt enthalten: Als Akt der Gesprächseröffnung zeigt sie nämlich, dass ich jemand bin, mit dem der Andere ins Gespräch kommen möchte. Vor jedem kommunikativen Austausch macht die Begrüßung daher deutlich, dass ich grüßenswert bin - das heißt, dass ich jemand bin, der überhaupt gegrüßt werden kann. Das Grüßen ist daher zuallererst ein Akt der Anerkennung: Der Andere erkennt mich als einen Jemand an, an den er seine Worte richtet.

Die Begrüßung kehrt damit eine Schicht der Ansprache hervor, die jeder Form des Sprechens eigen ist, denn: indem wir mit jemandem sprechen, sprechen wir mit jemandem. Das ist auch dann noch der Fall, wenn genau dieses semantisch verneint wird. Also etwa, wenn der Sprechende behauptet, dass der Adressat kein Jemand sei, sondern ein Tier, ein Ding oder schlichtweg inexistent. Selbst Anreden wie »Du Hund «, »Du Flasche« oder »Du Niemand « nämlich, die negieren, dass sie es mit Jemandem zu tun haben, wenden sich doch eben an diesen Jemand, um ihm zu sagen, dass er kein Jemand sei. ${ }^{7}$ Was abgesprochen werden soll, wird also zugleich vorausgesetzt, denn indem Einer zu einem Anderen spricht, erkennt er diesen als Jemanden an, der überhaupt angesprochen werden kann - sonst hätte der Sprachakt überhaupt keinen Sinn. Jedes Sprechen geht also mit einer minimalen Form der Anerkennung einher. Und das Grüßen als semantisch gereinigte Form des Sprechens macht diesen Umstand besonders gut deutlich - oder, um dem Gedanken eine andere Wendung zu geben: Wenn das Grüßen in erster Linie als Akt der Anerkennung zu verstehen ist, dann unterliegt das Grüßen vielleicht jeglichem Sprechen als eine fundamentale Schicht der Zugeselltheit an den Anderen.

Die Ansprache, die sich als gesprochenes Wort, als Blick oder auch als Geste realisieren kann, begründet unser soziales Dasein in fundamentaler Weise. ${ }^{8}$ Das

5 Vgl. das Stichwort >Grusz< in Jacob und Wilhelm Grimm: Deutsches Wörterbuch, Bd. 9, Leipzig 1971, S. 989-1016.

6 Vgl. Erving Goffman: »Der bestätigende Austausch«, in: ders., Das Individuum im ifffentlichen Austausch. Mikrostudien zur öffentlichen Ordnung, Frankfurt/Main 1982, S. 97-137, hier: S. 121.

7 "Wer Du spricht, hat kein Eiwas zum Gegenstand«, Martin Buber: Ich und Du, Stutlgurt 1995, S. 4: thnlich Avishai Margalit: Politik der Würde. Über Achtung

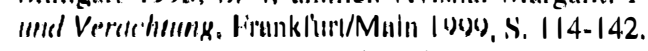

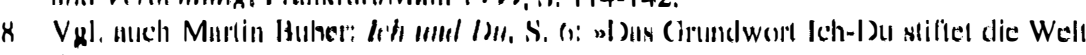
ilev' Iscoleluning." 
bedeutet zunächst: sie assoziiert mit anderen Menschen, sie vereinigt, verbindet. Durch den Akt der Anerkennung wird Gewissheit darüber erlangt, nicht nur nacktes Leben zu sein, sondern an einer Gemeinschaft zu partizipieren und ein Jemand im Kreis von Vielen zu sein. Dieser Gehalt ist auch in der Begriffsgeschichte des >Sozialen< eingelagert, die auf das lat. socius zurückgeht, das zugleich für den >Gefährten < als auch für die > Zugeselltheit $<$ zu und die >Teilnahme an diesem steht. Die schöpferische Kraft der Anrede hat zuerst Louis Althusser und im Anschluss an ihn auch Judith Butler hervorgehoben. Beide betonen, dass die Anerkennung, die der Ansprache eigen ist, ein Akt der Konstitution ist, in dem der Angesprochene allererst eine soziale Existenz erhält. ${ }^{9}$ Soziale Existenz zu erlangen, bedeutet darüber hinaus aber auch: situiert zu sein - wie es im indexikalischen Gehalt von Heideggers Begriff Da-sein zum Ausdruck kommt. Dazu-sein heißt: da, an dieser Stelle verortet zu sein. Und während Heidegger seinen Begriff des Daseins vor allem auf seine Situierung in Bezug auf zuhandenes Zeug denkt, steht der Begriff >soziales Dasein< hier für unsere Situierung gegenüber anderen ein. Angesprochen zu werden, bedeutet nicht nur in grundlegender Weise Teil einer menschlichen Gemeinschaft zu sein, sondern in dieser Gemeinschaft einen Platz einzunehmen zu sein. Denn selbst noch der belangloseste Gruß artikuliert durch seine Sprechweise eine Haltung des Ansprechenden, durch die wir unsere soziale Position erfahren - wie etwa geschätzt, geachtet oder geliebt zu sein. ${ }^{10}$

Das Angesprochenwerden stiftet so, um es mit einem Wort zu sagen, Geborgenheit - und zwar in dem zweifachen Sinn, der in diesem Begriff enthalten ist. Die Geborgenheit nennt einmal das Geborgenwerden im Sinne von >Emporheben $<$ und $>$ zur Erscheinung bringen $<$. Nicht jedoch als bloßes an die Oberfläche heben eines schon konstituierten Jemand, sondern im Sinne einer Hervorbringung: Der Akt der Ansprache macht den Angesprochenen, dadurch, dass er ihn als soziales Wesen anerkennt, allererst zu einem Jemand. Der zweite Sinn von Geborgenheit nennt die Grundbefindlichkeit, die mit dieser Bergung einhergeht: das Aufgehoben-Sein unter anderen. Indem mich der Andere anspricht, umhüllt er mich mit seiner Stimme und zeigt so auf fundamentale Weise seine Zuneigung. Unter der semantischen Oberfläche des Sprechens vollzieht sich so

9 Louis Althusser: »Ideologie und ideologische Staatsapparate (Anmerkungen für eine Untersuchung)«, in: ders., Ideologie und ideologische Staatsapparate. Aufsätze zur marxistischen Theorie, Hamburg/Westberlin 1977, S. 108-153; Judith Butler: Hass spricht. Zur Politik des Performativen, Berlin 1998, S. 41-47.

10 Natürlich kann das Grüßen auch exklusive Formen annehmen. Ein eindrückliches Beispiel ist der Hitlergruß. Der Gruß hat hier einerseits gemeinschaftsbildende Funktion: dadurch dass er die am Gespräch beteiligten unter der Obhut Hitlers zusammenschließt. Und andererseits erfüllt er zugleich auch eine ausschließende Aulgabe: Nicht nur, dass es Jüdinnen und Juden verboten war, den Hillergrulß als'rıführen, sondern auch, weil sich ihr Ausschluss aus dem >deulschen Kollekliv، im Gruß selbst symbolisierte und durch in vollzog. Vgl. dizu: Allert Tilmunn: Irer

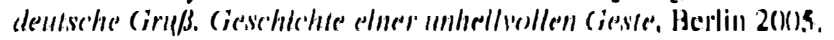

gleichsam ein intimer Akt: der Austausch von Wärme, Nähe und Zärtlichkeit. Daher sprechen wir auch oftmals nicht der Inhalte wegen, sondern um Willen jenes innigen Momentes, der dem Sprechen als Stiftung einer zwischenmenschlichen Geborgenheit innewohnt.

\section{Der Eigenname als Signum der Ansprache}

Im Eigennamen verdichtet sich die Stiftungskraft der Ansprache - bei der Einführung ins Soziale spielt er daher eine entscheidende Rolle. Das zeigt sich schon daran, dass die namentliche Vorstellung in Begrüßungszeremonien zumeist ein zentrales Ritual bildet. Die besondere Kraft der Ansprache mit dem Namen lässt sich an drei Punkten deutlich machen:

(i) Existenzwerdung als performativer Akt der Namensgebung: Die Namensgebung im Akt der Taufe gehört zu den von John Austin so genannten >ursprünglichen Performativa<, also jenen Klassen von Äußerungen, die dasjenige, was sie benennen, im selben Atemzug hervorbringen. Indem die Äußerung des Priesters »Hiermit taufe ich dich auf den Namen Felix Mendelsohn« dem Neugeborenen vor den Augen einer Gemeinschaft einen Namen verleiht, macht es dieses zu einem sozialen Wesen. ${ }^{11}$ Der Akt des Eintauchens in das Weihwasser kann in diesem Zuge als Versinnbildlichung des namentlichen Eintauchens in die soziale Gemeinschaft gelesen werden: Während der Nachname das Angesprochene in eine Kette von Vorfahren und damit in das Geflecht von sozialen Beziehungen einschreibt und unter diesen situiert, gibt der Vorname Einzigartigkeit und Individualität. Er nennt ein singuläres Wesen. Dieser Umstand wurde in der Onomastik etwa dadurch herausgestellt, dass Eigennamen zumeist weder einen Artikel haben noch in den Plural gesetzt werden können. Wir können zwar von >den Mendesohns< sprechen, aber wir sprechen normalerweise nicht von >der Felix< oder >den Felixen $<$. Das liegt daran, dass, was nur einem Individuum gehört, nicht in den Plural gesetzt und mit Artikeln verbunden werden kann, die ja die Funktion haben, aus einer Menge von Klassenmitgliedern ein Einzelnes herauszulösen. Die Verleihung des Eigennamens vereinzelt also auf fundamentale Weise - und zwar in dem Sinn, dass sie zu einem einzigartigen, sozialen Wesen macht. Diese soziale Prägekraft von Namen zeigt sich auch in

II Dass die Verleihung des Eigennamens die Zugehörigkeit zur sozialen Gemeinschaft verbül'gt, wie keine andere Form der Ansprache, macht ein Ritus der Kayan auf Borneo deutlich, von dem Diek, Bering berichtet: »dem Neugeborenen wird hier erst nach acht Monalen cill liggennamen verliehen. Stirbt es vor dem Ritual der Numeng(hung, so) wirl es genuu mil (len gleichen Riten hetrauert, die für eine Tot-

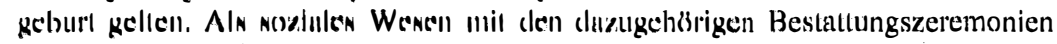

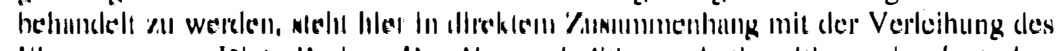

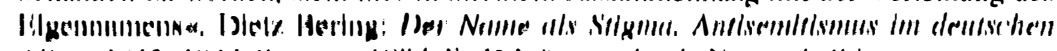

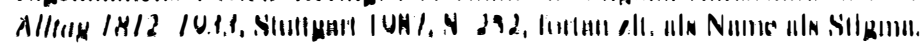


Schwellenriten, durch die wir in eine neue soziale Rolle schlüpfen. Der soziale Übergang etwa, der durch Eheschließung, den Einritt ins Kloster oder beim Amtsantritt eines Papstes stattfindet, wird jeweils durch einen neuen Namen besiegelt. Die Namensgebung steht hier für eine Art soziale Neugeburt, sie soll die soziale Verwandlung der betroffenen Person deutlich machen.

(ii) Verzeitlichung des sozialen Daseins zwischen Präsenz und Absenz: Durch den Eigennamen wird die Ansprache verzeitlicht und dauerhaft gemacht. Das wird an dem grundlegenden Unterschied zwischen der Ansprache mit einem $>$ Du $<$ und der namentlichen Ansprache deutlich: Die Ansprache mit einem $>$ Du $<$ hat keine kontinuierliche Referenz: >Du $<$ hat seine Kontinuität nur für die Dauer der Ansprache, denn in dem Moment, in dem eine andere Adressatin angesprochen wird, ist $>\mathrm{Du}<$ jemand anders. $>\mathrm{Du}<$, so lässt sich mit Benveniste sagen, ist ein >mobiles Zeichen<, dessen Referenz jeweils mit dem Vollzug des Sprechens zustande kommt - aufgrund seiner Indexikalität ist die benennende Kraft des Pronomens im Kontext verhaftet. ${ }^{12}$ Der Gebrauch des Eigennamens dagegen ist kontextübergreifend, seine Nennung nimmt nicht nur in ganz unterschiedlichen Situationen Bezug auf die adressierte Person, sondern ebenso in deren Abwesenheit - und genau darin liegt seine Besonderheit. Im Gegensatz zum > Du< handelt es sich beim Eigennamen also um ein >starres Zeichen $<$, dem seine Referenz anhaftet. ${ }^{13}$ Dadurch kann jederzeit, sogar bei Abwesenheit der betreffenden Person, auf die Existenz eines konkreten $>$ Du< Bezug genommen und damit das situationsbedingte Spiel von Präsenz und Absenz überschritten werden. Aus diesem Grunde stiftet der Eigenname wie keine andere Form der Benennung eine Verzeitlichung der sozialen Existenz.

(iii) Verstetigung der sozialen Existenz in der Zeit: Es war zunächst John Stuart Mill mit seiner realistischen und im Anschluss an ihn Saul Kripke mit seiner pragmatisch gewendeten Theorie des Eigennamens, die darauf hingewiesen haben, dass Eigennamen keine Bedeutung haben. ${ }^{14}$ Das bedeutet, dass sie keine begriffliche Aussage über die benannte Person machen und diese unter Absehung von konkreten Eigenschaften nennen. Dadurch ist der Name jedoch nicht das Wort mit der geringsten, sondern mit der umfassendsten Bedeutung: Er bezieht sich auf die Gesamtperson. Um den Namen auszufüllen, müssen wir keine Bedingungen erfüllen, wir müssen keine besondere Leistung erbringen oder erst in ihn hineinwachsen, er nennt uns einfach: jederzeit. Auch wenn sich unsere Eigenschaften in der Zeit verändern, der Eigenname nennt als Kind, als Erwachsener und im Greisenalter dieselbe Person. Unabhängig aller Ver-

12 Vgl. Emile Benveniste: »Die Natur der Pronomen«, in: ders., Probleme der allgemeinen Sprachwissenschaft, München 1974, S. 79-286, hier: S. 284.

13 Vgl. auch Saul A. Kripkes Begriff der >starren Bezeichnungsausdrücke` (rigid designators). Kripke: Name und Notwendigkeit, Frankfur//Main 1981, S. 59F.

14 John Stuart Mill: »Vom Namen«, in: Ursula Wolf (Hg.), Eigennemmen. Dokumentation einer Kontroverse, Frankfurt/Main 1985. S. 4.3-6.3; Kripke: Netmir

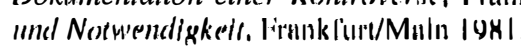

änderungen garantiert er, dass $>$ Ich $<$ gestern derselbe war, der $>$ Ich $<$ heute bin und $>$ Ich< morgen sein werde. Der Eigenname stiftet daher, wie es Paul Ricœur genannt hat, in besonderem Maße die >Selbigkeit< einer Person, ${ }^{15}$ seine Trägerschaft ist unabhängig von den unterschiedlichen Eigenschaften, die uns über die Zeit hinweg zukommen. Dadurch verleiht der Eigenname der sozialen Existenz auf einzigartige Weise Konstanz und Stabilität.

Fassen wir zusammen: Die Namensvergabe ist der sozial kodifizierte Stiftungsritus unserer sozialen Existenz, sie verleiht unserer sozialen Existenz ihren >sprachlichen Ankerpunkt $<.{ }^{16}$ Nicht zuletzt deshalb dient der Name in der Unterschrift als Identifikationszeichen: Er ist der Ausweis unserer Persönlichkeit, das Gesicht unseres sozialen Daseins, in der sich unsere gesamte soziale Existenz verdichtet. Die Ansprache mit dem Eigennamen ist die intimste Form der Ansprache. Und genau deswegen kann der Angriff auf den Eigennamen zu einem der gefäbrlichsten Angriffe auf unsere soziale Existenz werden.

\section{Dasein als Ausgesetztsein}

Unser Name ist uns zwar durch einen einmaligen Einsetzungsakt gegeben, doch muss er, um nicht in Vergessenheit zu geraten, immer wieder bekräftigt und erneuert werden. Daher ist die Geborgenheit, die durch den Namen gestiftet wird, immer prekär. Diese Prekarität wird dadurch gesteigert, dass wir uns unser soziales Dasein durch keinen selbstursprünglichen Stiftungsakt selbst verleihen können, denn es beruht fundamental auf einem Ausgesetztsein: Die soziale Existenz kommt immer vom Anderen her. Er ist die fortwährende Springquelle unserer sozialen Verfasstheit. Das hat seinen Grund in der Nicht-Reziprozität von Anrede und Antwort: Zwar kann einer einen Anderen ansprechen und sich dabei als soziales Wesen einzusetzen trachten, er kann seinen Namen nennen und darüber hinaus sogar behaupten, er sei eine wichtige soziale Persönlichkeit doch eine Antwort, die dieses Sprechen überhaupt als Sprechen anerkennt, kann ausbleiben. Denn zum Antwortregister auf solch eine Ansprache gehört nicht nur die verneinende Antwort, welche die Ansprache in Zweifel zu ziehen vermag, und dabei doch anerkennen würde, dass sie von jemandem getätigt wurde, sondern ebenso das Schweigen, welches das Ereignis der Antwort überhaupt auszusetzen vermag. So schreibt auch Bernhard Waldenfels: »Das Geben oder Verweigern einer Antwort vollzieht sich auf der Ebene des Aussageereignisses und nichl auf der Ebene des Aussagegehalts. ${ }^{17}$ Die verweigerte Antwort in Form des Scliweigens lässt den Ruf nach Anerkennung, der der Ansprache innewohnt, un-

1,5 Ponl Ric(xur: Dess sedbst als rill Anderere. Munchen 1996, S. 39-54.

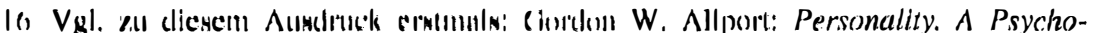

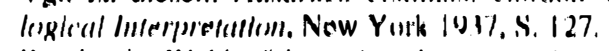

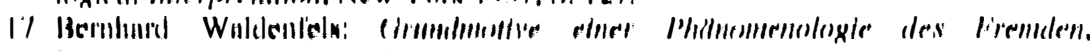

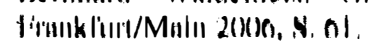


erwidert - sie erkennt nicht an, dass jemand gesprochen hat. In dieser Hinsicht ist man dem Anderen fundamental ausgeliefert: Denn erst wenn der Andere das Schweigen bricht und eine Antwort gibt, also seinerseits anspricht, anerkennt er wiederum den Anderen als soziales Wesen. Das selbst initiierte Sprechen, das in die soziale Existenz rufen soll, zielt in dieser Perspektive also immer auf eine Antwort, es ist ein Appell an den Anderen, seinerseits anzusprechen. Zwischen Anrede und Antwort existiert jedoch die Möglichkeit des Schweigens, welches das Wechselspiel von Anrede und Antwort unterbrechen kann. Als soziale Wesen existieren wir daher in einer grundsätzlichen Passivität: ausgesetzt an den Andern.

Im Ausgesetztsein gegenüber dem Anderen wurzelt eine fundamentale Form der Verletzungsoffenheit. Denn bevor wir das Risiko des Schweigens, in dem unser ganzes soziales Dasein auf dem Spiel steht, in Kauf nehmen, sind wir oftmals lieber bereit, eine demütigende soziale Situierung hinzunehmen. Das zeigt sich etwa in der Geschichte von einem jüdischen Mädchen, dass nach der Befreiung von einem alliierten Soldaten nach seinem Namen gefragt wird. Seine Antwort lautet: »Judensau«. Diese kurze Begebenheit kann sicherlich auf vielerlei Arten gelesen werden. Eine naheliegende Lesart wäre, dass sich hinter dieser Antwort die unmittelbar physische Existenzbedrohung durch die Nationalsozialisten verbirgt und dass das Mädchen die Beschimpfung als Namen aus Todesangst heraus angenommen hat. Neben dieser Lesart können wir die Geschichte jedoch auch als eine Allegorie auf das Begehren nach Anerkennung verstehen. Im Mittelpunkt der Geschichte steht dann, dass wir manchmal lieber verletzende Benennungen akzeptieren, als gar nicht adressiert zu werden. Das hat seinen Grund darin, dass es der Akt der Ansprache selbst ist, und nicht der semantische Gehalt, durch den wir als soziale Wesen anerkannt werden - und es ist genau dieser Umstand, durch den wir verletzungsoffen für diesen Gehalt sind. ${ }^{18}$ Anders ausgedrückt: Weil der semantische Gehalt der Ansprache im Angesicht des Ereignisses des Angesprochenwerdens nachrangig ist, sind wir für genau diesen Gehalt empfänglich. Auch wenn uns dieser an den sozialen Rand rückt, so erkennt er uns doch noch in einem grundlegenden Sinne als soziales Wesen an. Halten wir daher fest: Als soziale Wesen sind wir von der Adressierung durch andere abhängig. Auch wenn natürlich nicht alle Ad-

18 Auf diese Tatsache spielt auch Levinas an, wenn er schreibt: »Der Andere hält und bestätigt sich in seiner Andersartigkeit, sobald man ihn anruft, sei es auch nur, um ihm zu sagen, dass man mit ihm nicht sprechen kann, sei es, um ihn als Kranken einzustufen, um ihm sein Todesurteil anzukündigen. Während er ergriffen und verletzt wird, während ihm Gewalt geschieht, wird er gleichzeitig >geachtet..« Emmanuel Levinas: Totalität und Unendlichkeit, Freiburg 1993, S. 92f. Gank ähnlich hält auch Derrida fest: »Noch der schlimmste Folterknecht bezeugt das, was er an sich oder am Anderen zerstört, sofern er es nicht rettet, nämlich das Angesicht. Ob sie will oder nicht, die Feindlichkeit legt noch Zeugnis ah von der Citsulichkeil."

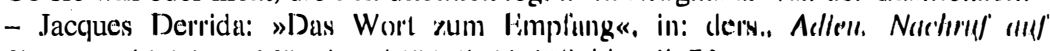

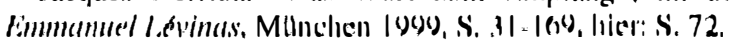

ressierungen das gleiche Gewicht haben und wir auf manche Formen der Ansprache sogar verzichten könnten, so wiederholt sich doch in jeder Ansprache im kleinen Maßstab das grundlegende Faktum unserer Ausgesetztheit an den Anderen. Und aufgrund dieser Ausgesetztheit halten wir oftmals lieber an verletzenden Ausdrücken und Namen fest, bevor wir gar nicht adressiert werden und unsere ganze soziale Existenz gefährdet ist.

Die Formen der Gewalt und der Verletzung, die derart ermöglicht werden, können sich nicht am Vorbild physischer Gewalt oder Verletzung orientieren. Denn sprachliche Gewalt operiert weder mit fassbaren, materiellen Waffen, noch hinterlässt sie sichtbare, materielle Wunden. Sprachliche Gewalt ist rein symbolisch, gerichtet ist sie nicht gegen die materiale, sondern gegen die soziale Existenz. Das schließt nicht aus, dass sie nicht auch körperliche Effekte zu zeitigen vermag; bedeutet aber in erster Line, dass sie in dem ihr eigenen Register der Gewaltförmigkeit und damit auch der ihr eigenen Form der Grausamkeit verstanden werden muss. Die Politik des Eigennamens gegenüber Juden und Jüdinnen ist dazu geeignet, beides deutlich hervortreten zu lassen.

\section{Politik des Eigennamens}

In der Geschichte des Antisemitismus wurde der jüdische Name auf einzigartige Weise zum Ziel einer Politik des Eigennamens. Zunächst noch scheinbar harmlos als Stichelei im Spott- und Schimpfnamen artikuliert, entfaltete sich später eine systematisch gelenkte Politik, die in der Ersetzung des Namens durch die Nummer im Lager gipfelte. Die Gewalt gegen den Namen war eine Gewalt gegen das soziale Dasein der Juden und Jüdinnen, eine Gewalt, in der letztlich sogar ihre Existenz als Ganze infragegestellt wurde. Die Politik des Eigennamens lässt sich dabei in drei Phasen einteilen: ${ }^{19}$

(i) Die >Taufe der deutschen Juden: Der entscheidende Einsatzpunkt der Politik des Eigennamens liegt im Jahr 1812. Bis zu diesem Datum waren die Juden in den deutschen Staaten fast vollkommen vom gesellschaftlichen Leben ausgeschlossen. Im Rahmen der Aufklärung wurde jedoch die Emanzipation der Juden vorangetrieben: Die erzwungene Absonderung sollte zugunsten einer Assimilation aufgehoben werden. Die Juden sollten ihre alte Identität hinter sich lassen und als Staatsbürger Teil der >deutschen Gesellschaft< werden. Diese soriale Neugeburt des >deutschen Juden< war unmittelbar mit einem Namens-

(1) Ier ()nomast Dietz Bering hat dic verschiedenen Stufen einer solchen Politik des ligennamens in mehreren Publikutionen hervorragend beschrieben. Der entscheidende: Umriss null hicr kur', wiedergegebon werden. Ausführlicher vgl. Dietz 13ering: Nelme "ls. S'tligmel: derw.: no iewull gegen Namen. Ein sprachwissenschaft-

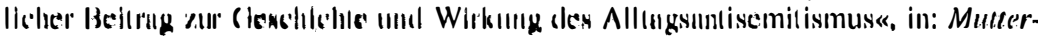

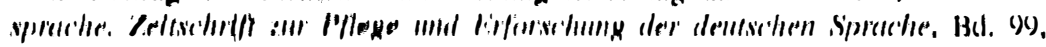

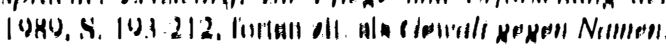


gebungsakt verbunden: Juden, die bis dahin traditionellerweise oftmals nur einen Rufnamen besaßen, sollten einen Familiennamen annehmen. In einer Art kollektivem Taufakt sollte durch den Namen die neue soziale Zugehörigkeit besiegelt werden - die Wahl des Namens sollte daher konsequenterweise auch keinen Beschränkungen unterliegen. Im Gegenteil: Juden wurden sogar ermutigt, germanische Namen anzunehmen, um sich ganz mit der deutschen Gesellschaft zu identifizieren. Damit hielt man sich ganz an das durch und durch aufklärerisch inspirierte Gutachten Wilhelm v. Humboldts zum Emanzipationsgesetz, im dem es hieß, dass es darum gehe, »dass jedem, der nicht in religiöser Hinsicht danach zu fragen hat, ungewiß bleibe, ob jemand Jude sey oder nicht.« (Gewalt gegen Namen, 198)

(ii) Restauration und einsetzende Regulierung: Spätestens mit der Entstehung des modernen Antisemitismus im späten 19. Jahrhundert - stellvertretend hierfür ist die Zeit zwischen den Erscheinungsdaten der antisemitischen >Gründungswerke< von Gobineau 1853 und Chamberlain $1899^{20}$ - wurde der Anfang des Jahrhunderts begonnene Emanzipationsprozess einzuschränken und rückgängig zu machen versucht. Antisemitische Proteste erhoben sich, dass in Bezug auf Namen wieder eindeutige Verhältnisse herzustellen seien. Doch diese Forderung war zum Scheitern verurteilt - denn ebenso, wie es Christen mit alttestamentarischen Namen gab, gab es nun auch Juden mit germanischen Namen. Dennoch wurde, um weitere >Veruneindeutigungen $<\mathrm{zu}$ stoppen, die Annahme deutscher Namen nach und nach strikten Regulierungen unterworfen. So wurde 1898 die freie Wahl der Vormamen für Juden zurückgenommen und schon amtlich eingetragene Assimilationsvornamen wurden wieder aberkannt. Zwei Jahre später, zur Jahrhundertwende wurden Namensänderungsgesuche von Personen >jüdischer Herkunft< unter besondere Handhabe gestellt und wiederum drei Jahre später wurde das Recht, anlässlich der christlichen Taufe einen weniger jüdisch geprägten Namen anzunehmen, genommen. Im gleichen Zuge, wie durch solche Beschränkungen versucht wurde, Juden die Annahme >deutscher Namen zu erschweren, sollten Deutsche zur Annahme germanischer Namen motiviert werden. So heißt es etwa in der Wochenzeitung Schlesische Landwacht in einer Ausgabe von 1924: »Wer Deutschland liebt, soll auch das Letzte wagen! Ein deutscher Mann muß deutschen Namen tragen. ${ }^{21}$

(iii) Markierung und Segregierung im NS: Die Nationalsozialisten setzen nach der Machtübergabe die bestehenden Bestrebungen fort: Nicht nur sollte es Deutschen mit scheinbar jüdischen Namen erleichtert werden, germanische Namen anzunehmen, sondern ebenso sollten, wie 1933 durch den Deutschen Anwaltsverein vorgeschlagen, alle jüdischen Namensänderungen wieder auf-

20 Arthur Gobineau: Versuch über die Ungleichheit der Menschenrussen (I85.3-55), .3 Bde., Stuttgart 1898-1901; Houston S. Chamberlain: Die (irumdlagen des X/X. Jahrhunderts (1890), 2 Bde.. Minchen 19)(9).

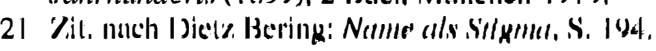

gehoben werden. In der Folge erscheint am 5. Januar 1938 tatsächlich das Gesetz über die »Änderung von Familiennamen und Vornamen«. Laut \$7 konnte nun eine Zwangsrückänderung eines jeden jüdischen Namenswechsels vor 1933 eingeleitet werden. Dieser Prozess hat sich jedoch als schwieriger und ungangbarer herausgestellt, als von den Nazis zunächst angenommen, sodass noch während das Verfahren der Zwangsrückänderung läuft, am 1. Januar 1939 die Bestimmung erscheint, dass jedem nicht deutlich erkennbaren jüdischen Namen die Beinamen >Israel< oder >Sara< zuzudiktieren seien. Dieser erzwungene Namensgebungsakt bildet in etwa das genaue Gegenstück zum Emanzipationsedikt von 1812: Ging es dort darum, die Juden in die deutsche Gesellschaft einzugliedern und sie als Juden unsichtbar zu machen, so sollte sie mit diesem zweiten >Taufakt $<$ in der Gesellschaft als Juden sichtbar gemacht werden, damit ihr Ausschluss umso reibungsloser vonstattengehen konnte. Dieser Prozess der Stigmatisierung wurde knapp zwei Jahre später noch einmal intensiviert. Durch die Einführung des Judensterns, auf dem in großen Lettern das Wort $>$ Jude $<$ prangte, waren Juden und Jüdinnen fortan einer permanenten Sichtbarkeit ausgesetzt: Das visuelle Stigma garantierte die Separierung noch vor jedem persönlichen Kontakt. Mit der Einschließung in Konzentrationslager und dem Einsetzen der Vernichtungspolitik begann schließlich das letzte Kapitel der nationalsozialistischen Namenspolitik: Bei der Ankunft im Lager wurde der Name durch die Ziffernkombination ersetzt, die alleine noch der Verwaltung der Vernichtung diente. Das Signum der sozialen Existenz wurde ersetzt durch die Nummer, das Zeichen des seriellen Todes.

\section{Beleldigen, Degradieren, Demütigen}

Durch die nationalsozialistische Politik des Eigennamens wurde auf ganz unterschiedliche Weise Gewalt durch Sprache gegen Juden und Jüdinnen ausgeübt. Je nach Missachtungsstrategie änderte sich dabei die Funktionsweise des Namens, sodass sich zunächst drei unterschiedliche Register einer Gewalt gegen den Namen unterscheiden lassen: Er wurde gewendet, zugeschrieben und entindividualisiert. $\mathrm{Zu}$ betonen ist, dass es sich dabei nicht immer um klar distinguierbare Praktiken handelt, sondern um ein Kontinuum, das sich in seiner Intensität immer weiter steigert und in dem die Übergänge nicht immer trennscharl verlaufen.

(i) Beleidigen (Namen wenden): Eine der nächstliegenden Formen der Gewall gegen Namen ist der Spottname. Durch ihn werden Namen so gewendet, dass cine Semantizităt zum Vorschein kommt. Im Unterschied zu einem schimplworl wie sudensalu jedoch, (las roh und brachial daher kommt, zehrt der Spontiname immer von cinem gew sisen Sprachwilz, durch welchen der Name

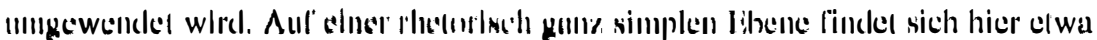

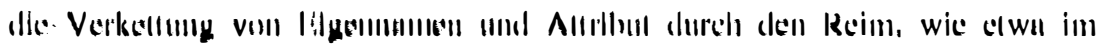


Spottnamen >Itzig-Witzig $<$. Die schon in diesem einfachen Sprachspiel angelegte Kreativität kann sich zu kunstvollen Metaphern steigern. Ein Beispiel dafür ist folgende Passage aus Wilhelm Marrs Aufruf »Wählet keine Juden« von 1873, in welchem scheinbar typisch jüdische Namen in ein darwinistisches Szenario überführt werden: »Wenn man in einem zoologischen Garten die Käfige öffnet und die Raubthiere losläßt, soll der Fuchs dann keine Hühner fressen, der Wolf keine Schafe zerreißen, der Löwe nicht in die Herden einbrechen, der Bär keinen Honig stehlen? Ja, sollen selbst Reh und Hirsch nicht nach Herzenslust weiden, wo sie gerade können? «(Gewalt gegen Namen, 200) ${ }^{22}$ Marr nimmt hier die Semantizität bestimmter Namen auf und überführt sie durch ein Verfahren der De- und Rekontextualisierung von ihrer rein kennzeichnenden Verwendungsweise in einen Kontext, in dem sie die sozialen Rollen ihrer Träger widerzuspiegeln scheinen. Marr bedient sich hier der Iterabilität des Sprechens: dass also kein sprachliches Zeichen in seiner Bedeutung ein für alle Mal abgeschlossen ist, sondern dass es durch Umschrift oder Aufpfropfung in neuen Kontexten neue Bedeutungen anzunehmen vermag. ${ }^{23}$ Diese Unabgeschlossenheit der sprachlichen Zeichen nutzt Marr, um scheinbar jüdische Namen in einen Kontext einzubetten, in dem diese plötzlich Eigenschaften über den Adressaten zu entbergen scheinen.

Doch kaum jemand würde auf die Idee kommen, solche Beleidigungen beim Wort zu nehmen. Denn bei den Spottnamen geht es nicht in erster Linie um den semantischen Gehalt, sondern um die kreative Wendung von Namen. Sie bildet den Grund, warum über Spottnamen gelacht wird. Entscheidend an der Beleidigung ist daher nicht so sehr, was sie auf einer semantischen Ebene aussagen, sondern welche sozialen Konstellationen durch sie geschaffen werden. Der Spottname schafft eine Solidargemeinschaft. Und zwar in dem Sinne, dass er diejenigen, die lachen, in diese Gemeinschaft einschließt und diejenigen, die lächerlich gemacht werden, aus dieser Gemeinschaft ausschließt. Das Beleidigen ist in diesem Sinne vor allem ein Unterscheiden von denjenigen, die dazugehören und denjenigen, die außen vor bleiben müssen. Der Spottnamen weist die adressierten Juden nicht nur darauf hin, dass sie nicht Teil der lachenden deutschen Gemeinschaft sind, er behauptet zugleich auch, dass ihre Existenz eine Andersartigkeit bedeutet. Die Beleidigung entpuppt sich so letztlich als ein Akt der Zurückweisung, der seine Adressaten als soziale Außenseiter markiert.

(ii) Degradieren (Namen zuweisen): Die Degradierung durch die $\mathrm{Zu}-$ schreibung von Namen erlangte durch den sogenannten Fall $>$ Weiß gegen

22 Ganz ähnlich arbeitet auch folgende Spottgeschichte: »Mit meinem Hund hab' ich e Zustand im Geschäft! Zuerst hatt' ich einen Kommis der hieß Katz, natürlich hat der Hund den Katz immer gebissen. Dann hab ich den Katz entlassen uncl einen genommen, der hieß Eckstein, da war's noch schlimmer. "[>Ficksteinc, der Stein un! den der Hund harnt] Ebd.. S. 196.

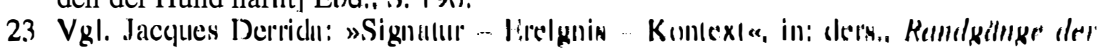

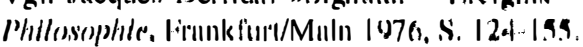

Goebbels< weithin Bekanntheit: ${ }^{24}$ Als Joseph Goebbels 1926 nach Berlin kommt und einen Protagonisten für seine antijüdische Propaganda sucht, fällt seine Wahl auf Bernhard Weiß, Jude aus assimiliertem Elternhaus, Träger des >Eisernen Kreuzes< und in den 1920ern Polizeivizepräsident von Berlin und damit Vorgesetzter der mit 20.000 Mann größten preußischen Behörde. Diese repräsentative Figur des öffentlichen Lebens dient Goebbels als Sinnbild für die >Unterwanderung< der angesehensten und mächtigsten Ämter Deutschlands durch >Ostjuden<. In seiner Zeitung Der Angriff versucht Goebbels Weiß als den paradigmatischen Fall der gefährlichen Mimikry-Natur der Juden zu enttarnen. Für seine Behauptung, dass hinter der vertrauenerweckenden Maske des germanischen >Bernhard $<$ alleine der >ewige Jude $<$ lauere, bedient sich Goebbels der Denunziation durch den Namen: Denn fortan wird er in seiner Anti-PolizeiKolumne >Vorsicht Gummiknüppel behaupten, der eigentliche Name von Bernhard Weiß sei Isidor. ${ }^{25}$ Schauen wir uns nun genauer an, was die $\mathrm{Zu}$ schreibung des Namens Isidor bedeutet: Der Name dient in der Verwendungsweise gegenüber Weiß nicht mehr als Eigenname für jemanden, sondern er versucht eine Aussage über diesen jemand zu machen. Eine Aussage, die an vielen anderen Stellen von Goebbels explizit gemacht wird (hässlich, stinkend, machtversessen, verschlagen etc.) und die sich im Namen Isidor dann zu einem Konglomerat aus Vorurteilen verdichtet. Der Name wird in diesem Fall also zur Zuschreibung: Das bedeutet, der Namen referiert nicht mehr auf eine Person unabhängig von ihren Eigenschaften, sondern er macht auf einer konnotativen Ebene eine Aussage über diese Person. Mit dem zur Aussage angeschwollenen Namen wird der Adressat nicht mehr als Einzelner benannt, vielmehr werden ihm Eigenschaften zugeschrieben - und in unserem Fall bedeutet das die typische Reihung der zeitgenössischen antisemitischen Stereotype. Die Zuschreibung von Namen vollzog sich dabei auf perfide Weise: Denn unabhängig davon, ob ihr (ichalt anerkannt wurde oder nicht, blieb sie am adressierten Subjekt hängen und sei es nur als Verdacht. Denn während gegen eine Zuschreibung wie etwa ,Betrüger< zumindest noch argumentiert werden kann, entzieht sich der Name weitgehend der Diskursivierbarkeit: Wo keine expliziten Zuschreibungen geguhen sind, kann ein Widerlegungsversuch keinerlei Ansatzpunkt finden. Auch wenn Weiß daher zwischen 1927 und 1932 sechzehn Mal gegen die Benennung durch Goebbels geklagt hatte, um seinen Namen reinzuwaschen, und auch wenn

24 Vgl. cluzu ausführlich: Dietz Bering: »Der Kampf um den Namen Isidor. Polizeivireprisicilent Bcrnharl Weißß gegen Gauleiter Joseph Goebbels«, in: Beiträge zur Nenme'nforscheng. 13c1. 18. 1983, S. 121-1.53.

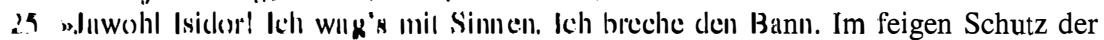

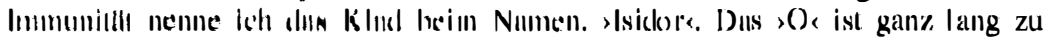

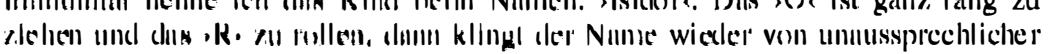

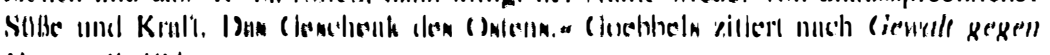

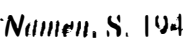


er dabei sogar den Zuspruch der Gerichte erhalten hatte, blieb sein Name dennoch beschmutzt.

Im Gegensatz zur Beleidigung, die durch die Markierung von NichtZugehörigkeit ihre Adressaten als soziale Außenseiten kenntlich machen sollte, funktioniert die Degradierung durch den Namen Isidor gerade dadurch, dass sie Weiß abwertende Eigenschaften zuschreibt und damit seine Autorität und Integrität infrage stellt. Der entscheidender analytische Unterschied zwischen Beleidigung und Demütigung liegt daher darin, dass während es jener in erster Linie darum geht die Betroffenen als soziale Außenseiter zu markieren, jene vor allem darauf abzielt eine soziale Minderwertigkeit zu behaupten. Die abweisende Distanzierung ist hier zugleich auch eine zugeschriebene Abwertung, weshalb die Degradierung aus dem Zweischritt von Zuweisen und Abwerten besteht.

(iii) Demütigen (Namen entindividualisieren): Von Akten der demütigenden Namensgebung wird schon im Rahmen des Gesetztes zur Emanzipation der Juden von 1812 berichtet. Während in Preußen Juden auf dieses Edikt hin ihren Nachnamen frei wählen konnten, hatten in Westgalizien die Juden ihren neuen Namen >in Empfang zu nehmen< - und oft war dieser voll Spott und Hohn: $>$ Trompetenschleim $<$, >Treppengeländer $<$ oder $>$ Knoblauchgeruch $<$ waren Namen, die in dieser Zeit verliehen wurden. Im Gegensatz zur Degradierung, bei der Namen begrifflichen Charakter erhalten, setzten diese Demütigungsakte genau auf den umgekehrten Mechanismus: Ein Begriff wird als Eigennamen eingesetzt. Dieser Mechanismus kommt auch über ein Jahrhundert später in der $>$ Judenpolitik < des Nationalsozialismus zum Einsatz: Ab dem 19.9.1941 hatten alle Juden an ihrer Kleidung deutlich sichtbar den Judenstern zu tragen, auf dem in an das Hebräische erinnernden Lettern das Worte $>$ Jude $<$ zu lesen war. Dieser Akt entspricht exakt jener paradigmatischen Namensgebungsszene, von der Wittgenstein in seinen Philosophischen Untersuchungen schreibt, dass sie darin bestehen »einem Ding ein Namenstäfelchen anzuheften. $\ll^{26}$ Der sechszackige Judenstern wurde nicht nur im ganz direkten Sinne angeheftet, sondern er diente zugleich auch als Name. Nur dass es sich hierbei eben um keinen echten Eigennamen handelte, sondern um einen Begriff für eine Klasse: Der Name eines Juden war fortan $>$ Jude $<$. Die individualitätsstiftende Kraft des Namens ging so zunehmend verloren, stattdessen machte der Judenstern seinen Träger zum Teil einer anonymen Masse. Und genau dieser Effekt war es, der von den Nazis intendiert war. Sie waren nicht mehr an der identitätsstiftenden Kraft der Benennung interessiert, sondern umgekehrt an der Zusammenfassung zu einer Klasse. Den sozialen Effekt dieses Ereignisses beschreibt Victor Klemperer in seiner Studie über die Sprache des >Dritten Reiches< folgendermaßen: »Welches war der schwerste Tag der Juden in den zwölf Höllen jahren? Nie habe ich von mir, nie von anderen eine andere Antwort erhalten als diese: der 19. September 1941.

26 Ludwig Wittgenstein: Philosophische Intersur'hungen. Frunk liurt/Muin 1971, 1.9. S. 221 .
Von da an war der Judenstern zu tragen. [...] nun war ich in jedem Augenblick für jeden kenntlich und durch die Kennzeichnung isoliert und vogelfrei. ${ }^{27}$ Der Judenstern war ein jederzeit sichtbares Stigma, auf dessen Verschleierung harte Strafen standen. Seine permanente Sichtbarkeit war die Voraussetzung für einen umfassenden Ausschluss von Juden und Jüdinnen aus dem öffentlichen Leben: Das Sitzen auf Parkbänken, der Gang ins Theater, die Benutzung der Straßenbahn und andere Formen der sozialen Teilhabe wurden durch ihn verunmöglicht. Der zudiktierte Name war ein Schlüssel zur Ausgrenzung der Juden aus dem alltäglichen sozialen Leben.

Die durch den Judenstern vollzogene Demütigung unterscheidet sich von der Degradierung in verschiedenen Hinsichten: Erstens wird die abwertende Kraft der Namensgebung durch ihre rechtliche Verankerung gesteigert. Sie ist nicht mehr allein auf individuelle oder kollektive Akte der Missachtung angewiesen, sondern wird durch den Staat souverän verfügt. Das Zuschreiben wird dadurch zu einem Zudiktieren. Zweitens steigerte sich die abwertende Kraft der Degradierung durch die staatliche Politik zur gänzlichen Entwertung. In dem Maße, wie Juden ihre Rechte immer weiter entzogen wurden, wurden sie als Menschen zweiter Klasse behandelt. Drittens verlor mit der Einführung des Judensterns der Namen seine individualitätsstiftende Funktion. Der Einzelne wurde zum Teil viner Masse, in der er keine individuelle Wertschätzung mehr genoss. Die entscheidenden Mechanismen, welche der Demütigung zugrunde liegen, bestehen (laher aus einem Dreischritt von Zudiktieren, Entwerten und Entindividualisieren.

\section{Entmenschlichung und sozialer Tod}

Wer bei der Ankunft an der Rampe nicht direkt in die Gaskammer kam, wurde im Lager unter unmenschlichen Bedingungen einer Vernichtung durch Arbeit uusgesetzt. Die dort vorherrschende Kommunikationsstruktur beschreibt Ruth Kliger in ihren autobiographischen Auseinandersetzungen folgendermaßen:

"Ich sollte diesen haßerfüllten Ton, der den Angesprochenen oder Angeschrieenen menschlich vertreibt und ihn oder sie gleichzeitig wie einen Gegenstand festhält, in den IIllchsten Wochen immer wieder hören und krümmte mich immer neu davor. Es war ein 'l'un. der nur darauf ausgerichtet war, einzuschüchtern und dadurch zu betäuben. Man Ilk'rkt meist nicht, wieviel Rücksicht im gewöhnlichen Gesprächston liegt [...] Man Nreilet mit seines Gleichen, wir waren nicht einmal Gegner. Das Autoritätsgebaren in Aurchiwilz war stets auf Aberkennung gerichtet, Ablehnung der menschlichen Existenz Iew Hillilings, seines oder ihres Rechts dazusein. ${ }^{28}$

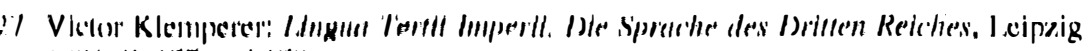

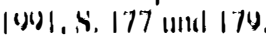

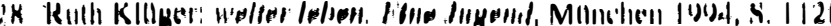


Die Aporie, in der diese Form der Ansprache mündet, stellt Klüger kurz darauf fest, wenn sie schreibt:

»Diese Spottsucht auf Kosten der Benachteiligten: Das kann nur meinen, dass es den Herrenmenschen doch nicht so selbstverständlich war, in den Todeslagern zu hantieren. Man musste sich durch schnoddrige Grausamkeit beweisen, dass diese Untermenschen keine Menschen waren. Und indem man es sich bewies, wurden sie wieder zu Menschen, denn man rechnete mit ihren Reaktionen auf den Spott. Der Spott wäre sinnlos gewesen ohne die Kränkung, auf die er abzielte. $\ll^{29}$

Die Situation der Ansprache im Lager stellt sich also folgendermaßen dar: Einerseits leugnet sie die Menschlichkeit ihres Gegenübers und andererseits muss sie im Akt der Leugnung dessen Menschlichkeit gerade anerkennen. Auch wenn die Ansprache noch so unmenschlich ist, bewahrt sie daher doch immer einen Rest an Menschlichkeit. Was daraus folgt, ist eine Spannung zwischen Erniedrigung und Entmenschlichung, in welcher etwa der Sozialphilosoph Avishai Margalit die Einzigartigkeit der nationalsozialistischen Demütigungen während der Shoa sieht. ${ }^{30}$

Dass diese Spannung aber bis zum Zerreißen gesteigert werden kann, zeigt sich nicht nur daran, dass die Lager-SS die Ansprache an die Häftlinge auf ein Minimum reduzierte, sondern vor allem an ihrem Umgang mit den Namen der Häftlinge. Die Auslöschung des Namens zugunsten der unter die Haut tätowierten Nummer bedeutete den Verlust jenes Ankerpunktes, in welchem das soziale Dasein situiert ist. Dieser Verlust kommt dem sozialen Tod unendlich nahe, denn die Nummer ist die Bezeichnung des Unmenschlichen, in ihr wird das soziale Dasein zur Sache. Die Zahl ist Stückzahl, sie dient der Quantifizierung, Abzählung und Aufrechnung, sie ist Teil einer alles Mögliche umfassenden universalen Serie. ${ }^{31}$ Dadurch wird eine Person als Ding angeeignet, die in einem bestimmten Kontext einen Funktionswert besitzt. Sie wird zum Teil einer Gleichung, in der unterschiedliche Größen miteinander verrechnet werden. Auch wenn die Nummer von ihrer identifizierenden Funktion daher dem Eigennamen gleichkommt, so hat sie doch einen ganz anderen sozialen Sinn. Wo der Name vermenschlicht, entmenschlicht die Nummer, denn während jener eine Zugehörigkeit zur menschlichen Gemeinschaft anzeigt, steht diese für die Bezeichnung des Nichtmenschlichen. Gestützt wurde die Erfahrung der Entmenschlichung dadurch, dass die zur Nummer herabgesunkenen Menschen tatsächlich so behandelt wurden, als wären sie Dinge. Das wird sehr deutlich am Tod, der im Lager gestorben wurde. In Claude Lanzmanns Dokumentation Shoa berichten Überlebende beispielsweise, dass ihnen die Deutschen verboten hatten,

29 Ebd., S. 143.

30 Avishai Margalit/Gabriel Motzkin: »The Uniqueness of the Holocinust«, in: P/llosophy and Public Affair. Bcl. 2.5. Hel' I, I096. S. 6.5-83.

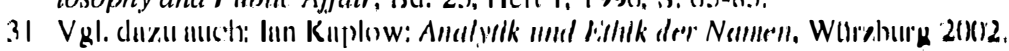

getötete Häftlinge als >Tote $<$ oder $>$ Opfer $<$ zu bezeichnen. Stattdessen mussten sie von >Figuren $<$, >Puppen $<$ oder $>$ Marionetten $<$ reden und die Ermordeten als Dinge adressieren. In den Lagern sollten keine Menschen mehr zu Grabe getragen, sondern nur noch Dinge vernichtet werden. Giorgio Agamben fasst diesen Prozess zusammen, wenn er schreibt: »In Auschwitz starb man nicht, es wurden Leichen produziert. Leichen ohne Tod, Nicht-Menschen, deren Umkommen zum Serienprodukt entwürdigt wurde. $\ll^{32}$ Der Tod war bar jeden sozialen Rituals menschlicher Trauerbekundung, keinerlei Zeremonie zeugte mehr vom Ableben eines Menschen - anstelle des individuellen Begräbnisses trat die Massenvernichtung von Stückzahlen. ${ }^{33}$

Man kann darüber streiten, ob die Nummer als technisch-administratives Hilfsmittel eingeführt wurde, um einen reibungsloseren Ablauf der Massentötung zu ermöglichen oder eine moralisch enthemmende Funktion für die Täter haben sollte, für die Betroffenen aber war sie unbestreitbar mit der Erfahrung der Aberkennung ihrer Menschlichkeit verbunden. Es ist genau dieser Punkt, durch den die Enteignung des Namens von einer Demütigung in eine Entwürdigung übergeht. Denn egal welche Ansprüche und Rechte wir in der menschlichen Würde begründet sehen, sie alle beruhen darauf, dass wir Menschen als Menschen behandeln. Die Entwürdigung aber tut genau das nicht mehr: Sie behandelt Menschen als Dinge. Auch wenn die Funktionsweise der Entwürdigung derjenigen der Demütigung sehr ähnlich ist, macht sie ihr gegenüber doch einen entscheidenden weiteren Schritt: Sie geht über die Entindividualisierung eines Menschen hinaus, indem sie diesem seine Menschlichkeit abspricht. In der Auslïschung des Namens wird dieser Schritt auf eindrückliche Weise vollzogen.

Der Verlust der Zugehörigkeit zur menschlichen Gemeinschaft bedeutete für die Betroffenen oftmals, dass keine positive Identifikation mit dem eigenen Selbst mehr möglich war. Sofern die Häftlinge unter den unmenschlichen Lagerhedingungen nicht noch untereinander, sei es auch einen noch so spärlichen sorialen Kreislauf aufrecht erhalten und so ihren Namen bewahren konnten - nicht umsonst wurden diejenigen, die im Lager überleben konnten und die von den ancleren Häftlingen gekannt und anerkannt waren, >Prominente< genannt -, traten dic Häftling in den Zustand des >verworfenen Lebens` ein, der den äußersten liluchtpunkt sozialer Ausschließungsprozesse bildet. Für die Inkarnation des verworfenen Lebens steht im Lager paradigmatisch die Figur des Muselmanns,

12. (iiorgio Agamben: Was von Auschwitz bleibt. Das Archiv und der Zeuge, l'runkl'url/Main 2003, S. 62, lortan zit. als Was von Auschwitz bleibt.

33 1) Burin liegl illch die Becleulung der Anstrengung, aus Nummern wieder Namen zu

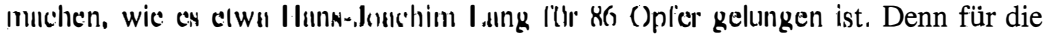

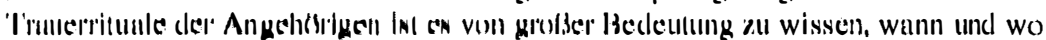

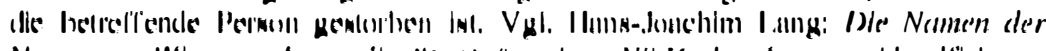

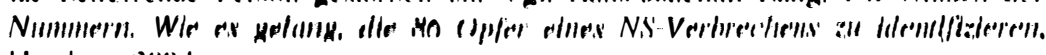

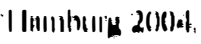


die häufig auch als »namenlose Gestalt« bezeichnet wurde. ${ }^{34}$ So charakterisiert wurden zunächst diejenigen Häftlinge, deren Erscheinungsbild von äußerster Auszehrung geprägt war. Ihr körperlicher Niedergang zeigte sich in Verfärbungen der Haut, dem Ausfall der Haare, der Bildung von Ödemen am ganzen Körper und der Anfälligkeit für jegliche Art von Infektionen. Bei ihrer Verelendung verloren die Betroffenen bis zu zwei Drittel ihres normalen Körpergewichts. Aufgrund ihrer körperlichen Gebrechlichkeit, so schreiben Zdzislaw Ryn und Stanislaw Klozińksi in ihrer bisher einzigartigen Studie, wurden die Muselmänner von allen Lagerakteuren gemieden:

»Der Muselmann weckte bei niemandem Mitleid und erfuhr von niemandem Herzlichkeit. Die Kameraden, deren Existenz ja selbst bedroht war, beachteten die Muselmänner nicht. Den Funktionshäftlingen brachten die Muselmänner zuviel Ärger ein, für die SSMänner waren sie unnützer Abfall. Die einen wie die anderen brachten sie um, jeder auf seine Weise. $\ll^{35}$

Der Muselmann stand für diejenige Figur, die aus allen sozialen Kreisläufen nahezu vollständig ausgeschlossen war und sich am Punkt äußerster sozialer Isolierung befand. In seiner Gestalt spiegelte sich ein Schicksal wieder, das alle Lagerinsassen bedrohte: der soziale Tod. Denn nachdem die anderen aufgehört hatten, den Muselmann als Mensch zu adressieren, verfiel dieser in völlige soziale Apathie. Sein Gesicht wurde teilnahmslos, der Blick trübe und seine Stimme immer leiser. Die Rede, die auf keine Antwort mehr hoffen konnte, wurde zum bloßen Gemurmel. Nicht nur soziale Ausdrucksfähigkeiten jedoch, auch elementare soziale Selbstpraktiken wie das Waschen oder der Gang auf die Toilette versiegten nach und nach - jegliche Einbettung in soziale Zusammenhänge löste sich nach und nach bis zur Gänze auf. In Berichten von Überlebenden wird der Muselmann daher lediglich noch als »Bündel physischer Funktionen « beschrieben, als ein »lebender Toter«, der an der »Schwelle zwischen Mensch und Nicht-Mensch « dahinvegetiert. ${ }^{36}$ In diesem Zustand sozialer Isolierung ging er zumeist unausweichlich seinem physischen Ende entgegen. Signifikant ist jedoch, dass gerade durch erneute Ansprache, sei es durch einen Brief von außen, eine auf rüttelnde Ansprache durch einen Kameraden oder eine menschliche Geste, der Muselmann manchmal vor seinem Schicksal gerettet

34 Vgl. dazu die wegweisenden Beiträgen von Wolfgang Sofsky: Die Ordnung des Terrors: Das Konzentrationslager, Frankfurt/Main 1997, S. 230 und Auschwitz.

35 Zdzislaw Ryn/Stanislaw Klodzińksi: »An der Grenze zwischen Leben und Tod. Eine Studie über die Erscheinung des Muselmanns im Konzentrationslager«, in: Hamburger Institut für Sozialforschung (Hg.): Auschwitz-Hefte. Bcl. I. Weinheim/Basel 1987, S. 89-154, hier: 127.

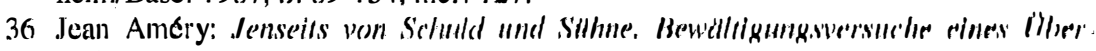

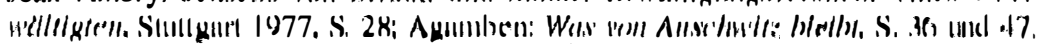

werden konnte. ${ }^{37}$ Das Minimum an menschlicher Anerkennung, das in solchen Gesten enthalten war, stiftete den Anfang eines sozialen Bandes, das den ausgemergelten Körpern die Kraft zum Überleben geben konnte.

Im Muselmann verkörpert sich das Grauen des Lagers auf einzigartige Weise, denn er ist der Unsagbare schlechthin: Nicht nur, dass seine namenlose Existenz von niemandem mehr angesprochen wurde, nicht nur, dass er selbst nicht mehr die Stimme erhob, vielmehr konnte auch nicht mehr über ihn gesprochen werden - denn vor dem im wörtlichen Sinn Unsagbaren versagt jegliche Sprache, wie Primo Levi feststellt: »Da merkten wir zum ersten mal, daß unsere Sprache keine Worte hat, diese Schmach zu äußern, dies Vernichten eines Menschen. ${ }^{38}$ Weil der Muselmann aus dem Kreislauf der Sprache ausgeschlossen ist, weil er jenseits der Grenzen der Sprache liegt, ist er grundlegend aus dem Bereich des Sagbaren ausgeschlossen. Und die Auslöschung des Namens bezeichnet genau jene Grenze, an dem dieser Ausschluss stattfindet und die Aporie der entmenschlichenden Ansprache zerbirst. Dass es sich hier um keine einfache Intensivierung und Steigerung, sondern einen kategorialen Überstieg handelt, wird daran deutlich, dass sich die Gewalt am Namen in und mit der Sprache vollzieht: durch Wenden, Zuweisen oder Entindividualisieren wurde der Eigenname zur Missachtung seiner Träger gebraucht. Der Entzug des Namens dagegen verstößt aus dem Bereich des Sagbaren: Mit seinem Verlust wird das sprachliche Band zerschnitten, verschwindet sein Träger aus dem Kreislauf der Sprache und wird zur Verkörperung des sozial Toten. Was sich in der (iestalt des Muselmanns inkarniert, ist ein namenloses Grauen.

\section{Literatur}

Agamben, Giorgio: Was von Auschwitz bleibt. Das Archiv und der Zeuge, Frankfurt/Main: Suhrkamp 2003.

Allport, Gordon W.: Personality. A Psychological Interpretation, New York: Henry Holt and Company 1937.

Althusser, Louis: »Ideologie und ideologische Staatsapparate (Anmerkungen für eine Untersuchung)«, in: ders., Ideologie und ideologische Staatsapparate. Aufsätze zur marxistischen Theorie, Hamburg/Westberlin: VSA 1977.

Améry, Jean: Jenseits von Schuld und Sühne. Bewältigungsversuche eines Überwelltigten, Stuttgart: Klett-Cotta 1977.

17 Vgl. die von Ryn Innd Kloclyińkssi gesammelten Zeugenaussagen: Zdzislaw Ryn/Stanislıw Klodrińksi: m/n der Girenze awischen Leben und Tod. Eine Studie

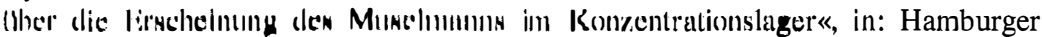

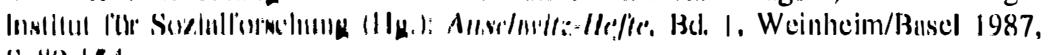
S. $Y(0)-1.94$.

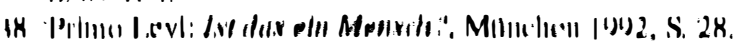


Benveniste, Emile: »Die Natur der Pronomen«, in: ders., Probleme der allgemeinen Sprachwissenschaft, München: List 1974, S. 279-286.

Bering, Dietz: »Der Kampf um den Namen Isidor. Polizeivizepräsident Bernhard Weiß gegen Gauleiter Joseph Goebbels«, in: Beiträge zur Namenforschung, Bd. 18, 1983.

- ders.: Der Name als Stigma. Antisemitismus im deutschen Alltag 1812-1933, Stuttgart: Klett-Cotta 1987.

- ders.: »Gewalt gegen Namen. Ein sprachwissenschaftlicher Beitrag zur Geschichte und Wirkung des Alltagsantisemitismus«, in: Muttersprache. Zeitschrift zur Pflege und Erforschung der deutschen Sprache, Bd. 99, 1989, S. 193-212.

Buber, Martin: Ich und Du, Stuttgart: Reclam 1995.

Butler, Judith: Hass spricht. Zur Politik des Performativen, Berlin: Berlin-Verlag 1998.

Chamberlain, Houston S.: Die Grundlagen des XIX. Jahrhunderts (1899), 2 Bde., München: Bruckmann 1919.

Derrida, Jacques: »Das Wort zum Empfang«, in: ders., Adieu. Nachruf auf Emmanuel Lévinas, München/Wien: Hanser 1999, S. 31-169.

- ders.: »Signatur - Ereignis - Kontext«, in: ders., Randgänge der Philosophie, Frankfurt/Main/Berlin/Wien: Ullstein 1976, S. 124-155.

Fruchtmann, Karl: Zeugen. Aussagen zum Mord an einem Volk, Köln: Kiepenheuer \& Witsch 1982.

Gobineau, Arthur: Versuch über die Ungleichheit der Menschenrassen (185355), 3 Bde., Stuttgart: Frommanns Verlag (E. Hauff) 1898-1901.

Goffman, Erving: »Der bestätigende Austausch «, in: ders., Das Individuum im öffentlichen Austausch. Mikrostudien zur öffentlichen Ordnung, Frankfurt/Main: Suhrkamp 1982, S. 97-137.

Grimm, Jacob/Grimm, Wilhelm: Deutsches Wörterbuch in 16 Bänden, Leipzig: Hirzel 1971.

Henneberg, Ilse (Hg.): Vom Namen zur Nummer. Einlieferungsritual in Konzentrationslagern, Bremen: Donat 1996.

Kaplow, Ian: Analytik und Ethik der Namen, Würzburg: Königshausen \& Neumann 2002.

Klemperer, Victor: Lingua Tertii Imperii. Die Sprache des Dritten Reiches, Leipzig: Reclam Leipzig 1991.

Klüger, Ruth: weiter leben. Eine Jugend, München: DTV 1994.

Kripkes, Saul A.: Name und Notwendigkeit, Frankfurt/Main: Suhrkamp 1981.

Lang, Hans-Joachim: Die Namen der Nummern. Wie es gelang, die 86 Opfer eines NS-Verbrechens zu identifizieren, Hamburg: Hoffmann und Campe 2004.

Levinas, Emmanuel: Totalität und Unendlichkeit, Freiburg: Alhor 1993.3.

Margalit, Avishai: Politik der Wilrde. "'here Achtung und Vercu'htum,e. Frunkfurt/Muin: Suhrkunp I)(s).
Mill, John Stuart: »Vom Namen«, in: Ursula Wolf (Hg.), Eigennamen Dokumentation einer Kontroverse, Frankfurt/Main: Suhrkamp 1985.

Ricœur, Paul: Das Selbst als ein Anderer, München/Freiburg: Fink 1996.

Ryn, Zdzislaw/Klodzińksi, Stanislaw: »An der Grenze zwischen Leben und Tod. Eine Studie über die Erscheinung des Muselmanns im Konzentrationslager«, in: Hamburger Institut für Sozialforschung (Hg.), Auschwitz-Hefte, Bd. 1, Weinheim/Basel: Beltz 1987.

Sofsky, Wolfgang: Die Ordnung des Terrors: Das Konzentrationslager, Frankfurt/Main: Fischer 1997.

Tilmann, Allert: Der deutsche Gruß. Geschichte einer unheilvollen Geste, Berlin Eichborn Verlag 2005.

Waldenfels, Bernhard: Grundmotive einer Phänomenologie des Fremden, Frankfurt/Main: Suhrkamp 2006

Wittgenstein, Ludwig: Philosophische Untersuchungen, Frankfurt/Main: Suhrkamp 1971.

Wolf, Ursula (Hg.): Eigennamen. Dokumentation einer Kontroverse, Frankfurt/Main: Suhrkamp 1985

' Zywulska, Krystina: Tanz, Mädchen ... Vom Warschauer Ghetto nach Auschwitz, München: DTV 1988. 